\title{
Existence and Uniqueness of Generalized Solutions to a Telegraph Equation with an Integral Boundary Condition via Galerkin's Method
}

\author{
Assia Guezane-Lakoud, ${ }^{1}$ Jaydev Dabas, ${ }^{2}$ \\ and Dhirendra Bahuguna ${ }^{3}$ \\ ${ }^{1}$ Laboratory of Advanced Materials, Badji Mokhtar University, P.O. Box 12, 23000 Annaba, Algeria \\ ${ }^{2}$ Department of Mathematics, Motilal Nehru National Institute of Technology Allahabad, \\ Allahabad 211 004, India \\ ${ }^{3}$ Department of Mathematics and Statistics, Indian Institute of Technology Kanpur, Kanpur 208 016, India \\ Correspondence should be addressed to Assia Guezane-Lakoud, a_guezane@yahoo.fr
}

Received 28 December 2010; Revised 1 March 2011; Accepted 4 March 2011

Academic Editor: Christian Corda

Copyright ( 2011 Assia Guezane-Lakoud et al. This is an open access article distributed under the Creative Commons Attribution License, which permits unrestricted use, distribution, and reproduction in any medium, provided the original work is properly cited.

We consider a telegraph equation with nonlocal boundary conditions, and using the application of Galerkin's method we established the existence and uniqueness of a generalized solution.

\section{Introduction}

The initial work devoted to the second-order partial differential equations with nonlocal integral conditions goes back to Cannon [1]. Subsequent investigations include [1-10] and many other references therein.

Nonlocal problems for some classes of partial differential equations have attracted much interest in the last few years, and several papers have been devoted to this subject. The problem of existence and uniqueness of solutions is of growing interest, as well as methods of obtaining explicit or approximate solutions for this kind of problems. The main reason of such a wide interest in this type of problems is that the non-local conditions occur in various equations of mathematical physics and in mathematical biology.

Nonlocal conditions come up when values of the function on the boundary are connected to values inside the domain. There are various types of non-local integral conditions for hyperbolic, parabolic, or elliptic equations; we point out an important non-local 
problem (see $[11,12])$, studied for a parabolic equation with the following non local conditions.

$$
\begin{aligned}
& u(0, t)=\int_{0}^{1} \alpha_{1}(x) u(x, t) d x+d_{1}(t) \\
& u(1, t)=\int_{0}^{1} \alpha_{2}(x) u(x, t) d x+d_{2}(t)
\end{aligned}
$$

connected with quasistationary thermoelasticity theory. The non-local boundary conditions of type

$$
\alpha(t)=\int_{0}^{1} u(x, t) d x, \quad u(1, t)=0
$$

arise in modeling the technology of integral circuits, studied in [13].

Problems with nonlocal integral conditions for multidimensional hyperbolic equations have been intensively studied in recent years. However, the nonlocal integral conditions for equations were in the form of second kind as

$$
\left.\frac{\partial u}{\partial \eta}\right|_{(x, t) \in \partial \Omega \times I}+\int_{0}^{T} \int_{\Omega} K(x, \xi, \tau) u(\xi, \tau) d \xi d \tau=0, \quad \forall x \in \partial \Omega,
$$

or

$$
\left.u(x, t)\right|_{(x, t) \in \partial \Omega \times I}+\int_{\Omega} K(x, \xi) u(\xi, t) d \xi=0, \quad \forall x \in \partial \Omega,
$$

where $\Omega$ is a bounded domain in $\mathbb{R}^{n}$ with a smooth boundary, $\partial u / \partial \eta$ is the inward normal vector to $\partial \Omega$; see $[14,15]$. Non-local integral conditions of first kind as

$$
\int_{\Omega} K(x, \xi) u(\xi, t) d \xi=0, \quad \forall x \in \partial \Omega
$$

are studied in [15]. Under some assumptions on the kernel $K$, existence and uniqueness of the generalized solution are established.

The present work deals with the application of the Galerkin method to determine a function $u=u(x, t), x \in \Omega \in \mathbb{R}^{n}$ is a bounded domain with a smooth boundary $\partial \Omega, t \in I=$ $(0, T)$, which satisfies, in weak sense, the telegraph equation

$$
l u=\frac{\partial^{2} u}{\partial t^{2}}+a \frac{\partial u}{\partial t}+b u-c \frac{\partial^{2} u}{\partial x^{2}}=f(x, t), \quad(x, t) \in Q=\Omega \times I,
$$


subject to the initial conditions

$$
\begin{aligned}
u(x, 0) & =\varphi(x), \\
\frac{\partial u}{\partial t}(x, 0) & =\psi(x)
\end{aligned}
$$

and the integral condition

$$
\left.\frac{\partial u}{\partial \eta}\right|_{(x, t) \in \partial \Omega \times I}+\int_{\Omega} K(x, \xi) u(\xi, t) d \xi=0, \quad \forall x \in \partial \Omega
$$

where $\partial u / \partial \eta$ is the inward normal vector to $\partial \Omega$. This equation arises in the analysis of propagation of electrical signals in a cable transmission line. Both the current and voltage satisfy an equation of this form. This equation arises also in the propagation of pressure waves in the study of pulsate blood flow in arteries.

Cannon et al. [5] have developed and analyzed finite-difference and finite-element Galerkin's methods for the solution of one-dimensional heat equation with the Neumann and integral conditions. Cannon and van der Hoek [4] presented numerical schemes based on the finite difference method. In [16], the authors have applied the Galerkin method to a parabolic problem with an integral boundary condition and established the existence, uniqueness, and continuous dependence upon the data of a weak solution. In [17-21], the authors have used the method of semidiscretization in time and have established the existence and uniqueness of a weak solution. In [22], the authors have discussed some existence uniqueness results for a neutral functional differential equation with a nonlocal initial condition via the Galerkin approximation.

The paper is organized as follows. In Section 2, we specify notations, state some inequalities and make the sense of the desired solution precise. In Section 3, we establish the uniqueness of the solution. Finally, Section 4, is devoted to the construction of the approximate solution and its existence via the Galerkin method.

\section{Notation and Definition}

Let $L^{2}(Q)$ be the usual space of Lebesgue square integrable real functions on $Q$ whose inner product and norm will be denoted by $($, $)$ and $\|\cdot\|$, respectively.

$W^{1,2}(Q)$ is the Sobolev space consisting of functions such that all derivatives lower than one belong to $L^{2}(Q)$ equipped with the norm

$$
\|u\|_{W^{1,2}(Q)}^{2}=\|u\|^{2}+\|\nabla u\|^{2}+\left\|u_{t}\right\|^{2}
$$

We define the space $W_{T}^{1,2}(Q)=\left\{v(x, t) \in W^{1,2}(Q), v(x, T)=0\right\}$. 


\subsection{Some Useful Inequalities: Gronwall Inequalities [23]}

Let $h(t)$ and $y(t)$ be two integrable nonnegative functions on the interval $I$ with $h(t)$ nondecreasing. If

$$
y(t) \leq h(t)+c \int_{0}^{t} y(s) d s, \quad \forall t \in I,
$$

where $c$ is a positive constant, then $y(t) \leq h(t) e^{c t}, \forall t \in I$.

Cauchy Schwarz inequality:

$$
\left(\int f(t) g(t) d t\right)^{2} \leq\left(\int|f(t)|^{2} d t\right)\left(\int|g(t)|^{2} d t\right)
$$

$\varepsilon$-Cauchy inequality:

$$
|\alpha \beta| \leq \frac{\varepsilon}{2} \alpha^{2}+\frac{1}{2 \varepsilon} \beta^{2}, \quad \forall \alpha, \beta \in \mathbb{R}, \forall \varepsilon \in \mathbb{R}_{+}^{*} .
$$

Trace inequality:

$$
\int_{\partial \Omega}|v|^{2} d s d t \leq \int_{\Omega}\left(\varepsilon|\nabla v|^{2}+c(\varepsilon)|v|^{2}\right) d x d t
$$

where $c(\varepsilon)$ is a positive constant that depends only on $\varepsilon$ and on the domain $\Omega$.

\subsection{Definition of Generalized Solution}

We define the generalized solution of the problem (1.6)-(1.8) as a weak solution that satisfies for all $v \in W_{T}^{1,2}(Q)$

$$
(l u, v)=(f, v) .
$$


Integrating by parts, we get

$$
\begin{aligned}
\int_{Q} u_{t t}(x, t) v(x, t) d x d t & =-\int_{\Omega} \psi(x) v(x, 0) d x-\int_{Q} u_{t}(x, t) v_{t}(x, t) d x d t \\
\int_{Q} u_{t}(x, t) v(x, t) d x d t & =-\int_{\Omega} \varphi(x) v(x, 0) d x-\int_{Q} u(x, t) v_{t}(x, t) d x d t \\
\int_{Q} u_{x x}(x, t) v(x, t) d x d t= & -\int_{0}^{T} \int_{\partial \Omega} v\left(\int_{\Omega} K(x, \xi) u(\xi, t) d \xi\right) d s d t \\
& -\int_{Q} \nabla u(x, t) \nabla v(x, t) d x d t
\end{aligned}
$$

Regrouping the identities (2.7), then (2.6) becomes

$$
\begin{gathered}
\int_{Q}\left(c \nabla u \nabla v-u_{t} v_{t}-a u v_{t}+b u v\right) d x d t+c \int_{0}^{T} \int_{\partial \Omega} v\left(\int_{\Omega} K(x, \xi) u(\xi, t) d \xi\right) d s d t \\
=\int_{Q} f v d x d t+\int_{\Omega} \psi(x) v(x, 0) d x+a \int_{\Omega} \varphi(x) v(x, 0) d x
\end{gathered}
$$

Definition 2.1. By a generalized solution of problem (1.6)-(1.8) one means a function $u \in W^{1,2}(Q)$ such that the identity $(2.8)$ holds for all function $v \in W_{T}^{1,2}(Q)$.

\section{Uniqueness of the Generalized Solution}

Now we will show that the generalized solution of problem (1.6)-(1.8), if it exists, is unique.

Theorem 3.1. Assume that $\varphi \in W^{1,2}(\Omega), \psi \in L_{2}(\Omega), f \in L_{2}(Q), K(x, y) \in C(\Omega \times \Omega)$, and

$$
\max _{\bar{Q}}|K(x, y)| \leq k_{1}
$$

then the generalized solution of problem (1.6)-(1.8), if it exists, is unique.

Proof. Suppose that there exist two different generalized solutions $u_{1}$ and $u_{2}$ for the problem (1.6)-(1.8); then obviously their difference $u=u_{1}-u_{2}$ is a generalized solution of the problem (1.6)-(1.8) with homogeneous equation and homogeneous initial and non local conditions, that is, $f=\varphi=\psi=0$. We will prove that $u=0$ in $Q$. Let $v \in W_{T}^{1,2}(Q)$, and denote $Q^{\tau}=$ $\{(x, t) ; 0<x<1,0<t \leq \tau \leq T\}$. Consider the function

$$
v(x, t)= \begin{cases}\int_{t}^{\tau} u(x, \theta) d \theta, & 0 \leq t \leq \tau, \\ 0, & \tau \leq t \leq T .\end{cases}
$$


The identity (2.8) becomes

$$
\int_{Q}\left(c \nabla u \nabla v-u_{t} v_{t}-a u v_{t}+b u v\right) d x d t+c \int_{0}^{T} \int_{\partial \Omega} v\left(\int_{\Omega} K(x, \xi) u(\xi, t) d \xi\right) d s d t=0
$$

Substituting $v$ into (3.3), integrating by parts, and then using the fact that $v_{t}(x, t)=-u(x, t)$, it follows that

$$
\begin{aligned}
\int_{\Omega}\left(c(\nabla v(x, 0))^{2}+u^{2}(x, \tau)+b v^{2}(x, 0)\right) d x= & -2 c \int_{0}^{\tau} \int_{\partial \Omega} v\left(\int_{\Omega} K(s, \xi) u(\xi, t) d \xi\right) d s d t \\
& +2 a \int_{0}^{\tau} \int_{\Omega} u^{2}(x, t) d x d t .
\end{aligned}
$$

Using the assumption on the function $K$, we get

$$
\begin{aligned}
& \int_{\Omega}\left((\nabla v(x, 0))^{2}+u^{2}(x, \tau)+v^{2}(x, 0)\right) d x \\
& \quad \leq C_{1}\left(\int_{0}^{\tau} \int_{\partial \Omega}|v|\left(\int_{\Omega}|u(\xi, t)| d x\right) d s d t+\int_{0}^{\tau} \int_{\Omega} u^{2}(x, t) d x d t\right),
\end{aligned}
$$

where $C_{1}=\max \left(2 c k_{1}, 2 a\right) / \min (c, 1, b)$. Now, applying Cauchy Schwarz inequality to the first term in the right-hand side of (3.5), then $\varepsilon$-Cauchy inequality with $\varepsilon=1$, yields

$$
\int_{\Omega}\left((\nabla v(x, 0))^{2}+u^{2}(x, \tau)+v^{2}(x, 0)\right) d x \leq C_{1}\left(\int_{0}^{\tau} \int_{\partial \Omega}|v|^{2} d s d t+2 \int_{0}^{\tau} \int_{\Omega} u^{2}(x, t) d x d t\right) .
$$

Using the trace inequality, we obtain

$$
\begin{aligned}
& \int_{\Omega}\left((\nabla v(x, 0))^{2}+u^{2}(x, \tau)+v^{2}(x, 0)\right) d x \\
& \quad \leq C_{1}\left(\int_{0}^{\tau} \int_{\Omega}\left(\epsilon(\nabla v)^{2}+c(\varepsilon) v^{2}\right) d x d t+2 \int_{0}^{\tau} \int_{\Omega} u^{2}(x, t) d x d t\right) .
\end{aligned}
$$

Considering the function

$$
w(x, t)=\int_{0}^{t} u(x, \theta) d \theta
$$


it is easy to see that $v(x, t)=w(x, \tau)-w(x, t), \nabla v(x, 0)=\nabla w(x, \tau)$, and $(\nabla v(x, t))^{2} \leq$ $2(\nabla w(x, \tau))^{2}+2(\nabla w(x, t))^{2}$. Consequently, substituting $w$ in (3.7), we get

$$
\begin{aligned}
\int_{\Omega}\left((\nabla w(x, \tau))^{2}+w^{2}(x, \tau)+u^{2}(x, \tau)\right) d x \leq & 2 \tau C_{1} \int_{\Omega}\left(\epsilon(\nabla w(x, \tau))^{2}+c(\epsilon) w^{2}(x, \tau)\right) d x \\
& +2 C_{1} \int_{0}^{\tau} \int_{\Omega}\left(\epsilon(\nabla w)^{2}+c(\epsilon) w^{2}+u^{2}\right) d x d t
\end{aligned}
$$

Seting

$$
C_{2}=2 C_{1} \max (\epsilon, c(\epsilon)), \quad C_{3}=2 C_{1} \max (\epsilon, c(\epsilon), 1),
$$

it follows that

$$
\begin{aligned}
\int_{\Omega}\left((\nabla w(x, \tau))^{2}+w^{2}(x, \tau)+u^{2}(x, \tau)\right) d x \leq & \tau C_{2} \int_{\Omega}\left((\nabla w(x, \tau))^{2}+w^{2}(x, \tau)\right) d x \\
& +C_{3} \int_{0}^{\tau} \int_{\Omega}\left((\nabla w)^{2}+w^{2}+u^{2}\right) d x d t
\end{aligned}
$$

Since $\tau$ is arbitrary chosen, let $1-\tau C_{2}>0$; then (3.11) becomes

$$
\left(1-\tau C_{2}\right) \int_{\Omega}\left((\nabla w(x, \tau))^{2}+w^{2}(x, \tau)+u^{2}(x, \tau)\right) d x \leq C_{3} \int_{0}^{\tau} \int_{\Omega}\left((\nabla w)^{2}+w^{2}+u^{2}\right) d x d t .
$$

Applying Gronwall Lemma, we get

$$
\left.\int_{\Omega}\left((\nabla w(x, \tau))^{2}+w^{2}(x, \tau)+u^{2}(x, \tau)\right) d x \leq 0, \quad \forall \tau \in\right] 0, \frac{1}{C_{2}}[
$$

Consequently, we obtain $u(x, \tau)=0$, for all $x \in \Omega$ and $\tau \in] 0,1 / C_{2}[$.

If $T \leq 1 / C_{2}$, then $u=0$ in $Q$. In the case where $T \geq 1 / C_{3}$, we see that $] 0, T$ [C $\left.\cup_{n=1}^{n=n_{0}}\right](n-1) / C_{2}, n / C_{2}\left[\right.$, where $n_{0}=\left[C_{2} T\right]+1,\left[C_{2} T\right]$ is the entire part of $C_{2} T$; then repeating the preceding reasoning for $\tau \in](n-1) / C_{2}, n / C_{2}[$, we get $u(x, \tau)=0$, for all $\tau \in](n-$ $1) / C_{2}, n / C_{2}[$, and then $u(x, t)=0$ in $Q$. Thus, the uniqueness is proved.

Remark 3.2. It should be noted that we can prove the uniqueness theorem by using a priori estimates, so we establish that the generalized solution, if it exists, satisfies the inequality

$$
\|u\|_{W^{1,2}(Q)}^{2} \leq C\left[\|f\|^{2}+\|\psi\|_{L_{2}(\Omega)}^{2}+\|\varphi\|_{W^{1,2}(\Omega)}^{2}\right] .
$$




\section{Existence of Generalized Solution}

Theorem 4.1. Assume that the assumptions of Theorem 4.1 hold; then the non-local problem (1.6)(1.8) has a unique solution $u \in W^{1,2}(Q)$.

Proof. In order to prove the existence of the generalized solution we apply Galerkin's method. Let $\left\{w_{k}(x)\right\}$ be a fundamental system in $W^{1,2}(\Omega)$, such that $\left(w_{k}, w_{i}\right)_{L_{2}(Q)}=\delta_{k, i}$. Now we will try to find an approximate solution of the problem (1.6)-(1.8) in the form

$$
u^{(n)}=\sum_{k=1}^{n} \alpha_{k}(t) w_{k}(x)
$$

The approximates of the functions $\varphi(x)$ and $\psi(x)$ are denoted, respectively, by

$$
\begin{gathered}
\varphi^{(n)}(x)=\sum_{k=1}^{n} \varphi_{k} w_{k}(x), \quad \psi^{(n)}(x)=\sum_{\sum_{k=1}}^{n} \psi_{k} w_{k}(x), \\
\alpha_{k}(0)=\varphi_{k}, \quad \alpha_{k}^{\prime}(0)=\psi_{k} .
\end{gathered}
$$

Substituting the approximation solution in (1.6), multiplying both sides by $w_{i}(x)$, and then integrating according to $x$ on $\Omega$, we get

$$
\begin{aligned}
& \int_{\Omega}\left(c \nabla u^{(n)} \nabla w_{i}+u_{t t}^{(n)} w_{i}+a u_{t}^{(n)} w_{i}+b u^{(n)} w_{i}\right) d x \\
& \quad+c \int_{\partial \Omega} w_{i}\left(\int_{\Omega} K(x, \xi) u^{(n)}(\xi, t) d \xi\right) d s=\int_{\Omega} f w_{i} d x .
\end{aligned}
$$

Substituting (4.1) in (4.3), we obtain

$$
\begin{aligned}
& \int_{\Omega} \sum_{k=1}^{n}\left(c \alpha_{k} \nabla w_{k} \nabla w_{i}+\alpha_{k}^{\prime \prime} w_{k} w_{i}+a \alpha_{k}^{\prime} w_{k} w_{i}+b \alpha_{k} w_{k} w_{i}\right) d x \\
& \quad+c \int_{\partial \Omega} \sum_{k=1}^{n} w_{i}\left(\alpha_{k} \int_{\Omega} K(x, \xi) w_{k}(\xi) d \xi\right) d s=\int_{\Omega} f w_{i} d x .
\end{aligned}
$$

This implies that

$$
\begin{aligned}
& \sum_{k=1}^{n}\left[\alpha_{k}^{\prime \prime}\left(w_{k}, w_{i}\right)_{L_{2}(\Omega)}+\alpha_{k}^{\prime}\left(a w_{k}, w_{i}\right)_{L_{2}(\Omega)}+\alpha_{k}\left(\left(c \nabla w_{k}, \nabla w_{i}\right)_{L_{2}(\Omega)}+\left(b w_{k}, w_{i}\right)_{L_{2}(\Omega)}\right)\right] \\
& +\sum_{k=1}^{n} \alpha_{k} \int_{\partial \Omega} c w_{i}\left(\int_{\Omega} K(x, \xi) w_{k}(\xi) d \xi\right) d s=\int_{\Omega} f w_{i} d x
\end{aligned}
$$


Denote

$$
\begin{aligned}
\beta_{k, i} & =\left(c \nabla w_{k}, \nabla w_{i}\right)_{L_{2}(\Omega)}+\left(b w_{k}, w_{i}\right)_{L_{2}(\Omega)}+\int_{\partial \Omega} c w_{i}\left(\int_{\Omega} K(x, \xi) w_{k}(\xi) d \xi\right) d s \\
f_{i} & =\int_{\Omega} f w_{i} d x
\end{aligned}
$$

Then (4.5) becomes

$$
\sum_{k=1}^{n}\left[\alpha_{k}^{\prime \prime} \delta_{k, i}+a \alpha_{k}^{\prime} \delta_{k, i}+\alpha_{k} \beta_{k, i}\right]=f_{i}
$$

We obtain a system of differential equations of second order according to the variable $t$ with smooth coefficients and the initial conditions

$$
\alpha_{k}(0)=\varphi_{k}, \quad \alpha_{k}^{\prime}(0)=\psi_{k}
$$

Consequently, we get a Cauchy problem of linear differential equations with smooth coefficients that is uniquely solvable. So it has a unique solution $u^{(n)}$ satisfying (4.3).

Lemma 4.2. The sequence $\left(u^{(n)}\right)$ is bounded.

Proof. Multiplying (4.4) by $\alpha_{i}^{\prime}$ and summing over $i$ from 1 to $n$, we get

$$
\begin{aligned}
& \int_{\Omega}\left(u_{t}^{(n)} u_{t t}^{(n)}+c \nabla u^{(n)} \nabla u_{t}^{(n)}+a u_{t}^{(n)} u_{t}^{(n)}+b u^{(n)} u_{t}^{(n)}\right) d x \\
& \quad+c \int_{\partial \Omega} u_{t}^{(n)}\left(\int_{\Omega} K(x, \xi) u^{(n)}(\xi, t) d \xi\right) d s=\int_{\Omega} f u_{t}^{(n)} d x .
\end{aligned}
$$

Integrating by parts the left-hand side of $(4.9)$ over $t$ on $(0, \tau)$ yields

$$
\begin{aligned}
\int_{0}^{\tau} \int_{\Omega} u_{t}^{(n)} u_{t t}^{(n)} d x d t= & \frac{1}{2} \int_{\Omega}\left[\left(u_{t}^{(n)}(x, \tau)\right)^{2}-\left(u_{t}^{(n)}(x, 0)\right)^{2}\right] d x, \\
\int_{0}^{\tau} \int_{\Omega} \nabla u^{(n)} \nabla u_{t}^{(n)} d x d t= & \frac{1}{2} \int_{\Omega}\left[\left(\nabla u^{(n)}(x, \tau)\right)^{2}-\left(\nabla u^{(n)}(x, 0)\right)^{2}\right] d x, \\
\int_{0}^{\tau} \int_{\Omega} u^{(n)} u_{t}^{(n)} d x d t= & \frac{1}{2} \int_{\Omega}\left[\left(u^{(n)}(x, \tau)\right)^{2}-\left(u^{(n)}(x, 0)\right)^{2}\right] d x \\
\int_{0}^{\tau} \int_{\partial \Omega} u_{t}^{(n)}\left(\int_{\Omega} K(x, \xi) u^{(n)}(\xi) d \xi\right) d s d t= & \int_{\partial \Omega} u^{(n)}(x, \tau)\left(\int_{\Omega} K(x, \xi) u^{(n)}(\xi, \tau) d \xi\right) d s \\
& -\int_{\partial \Omega} u^{(n)}(x, 0)\left(\int_{\Omega} K(x, \xi) u^{(n)}(\xi, 0) d \xi\right) d s \\
& -\int_{0}^{\tau} \int_{\partial \Omega} u^{(n)}\left(\int_{\Omega} K(x, \xi) u_{t}^{(n)}(\xi) d \xi\right) d s d t .
\end{aligned}
$$


Substituting the four last identities into (4.9), we get

$$
\begin{aligned}
& \int_{\Omega}\left[\left(u_{t}^{(n)}(x, \tau)\right)^{2}+c\left(\nabla u^{(n)}(x, \tau)\right)^{2}+b\left(u^{(n)}(x, \tau)\right)^{2}\right] d x \\
& =\int_{\Omega}\left[\left(u_{t}^{(n)}(x, 0)\right)^{2}+c\left(\nabla u^{(n)}(x, 0)\right)^{2}+b\left(u^{(n)}(x, 0)\right)^{2}\right] d x \\
& \quad-2 c \int_{\partial \Omega} u^{(n)}(x, \tau)\left(\int_{\Omega} K(x, \xi) u^{(n)}(\xi, \tau) d \xi\right) d s \\
& \quad+2 c \int_{\partial \Omega} u^{(n)}(x, 0)\left(\int_{\Omega} K(x, \xi) u^{(n)}(\xi, 0) d \xi\right) d s \\
& \quad+2 c \int_{0}^{\tau} \int_{\partial \Omega} u^{(n)}(x, t)\left(\int_{\Omega} K(x, \xi) u_{t}^{(n)}(\xi, t) d \xi\right) d s d t \\
& \quad-2 a \int_{0}^{\tau} \int_{\Omega}\left(u_{t}^{(n)}\right)^{2} d x d t+2 \int_{0}^{\tau} \int_{\Omega} f u_{t}^{(n)} d x d t .
\end{aligned}
$$

Applying Cauchy Schwarz inequality, $\epsilon$-Cauchy inequality, the hypothesis on the operator $K$, and the fact that $-2 a \int_{0}^{\tau} \int_{\Omega}\left(u_{t}^{(n)}\right)^{2} d x d t \leq 0$ to the second term in the right hand side of (4.11), we get

$$
\begin{aligned}
& \left|\int_{\partial \Omega} u^{(n)}(x, \tau)\left(\int_{\Omega} K(x, \xi) u^{(n)}(\xi, \tau) d \xi\right) d s\right| \\
& \quad \leq \frac{1}{2}\left(c(\varepsilon)+k_{1}^{2}|\partial \Omega|\right) \int_{\Omega}\left(u^{(n)}(x, \tau)\right)^{2} d x+\frac{\varepsilon}{2} \int_{\Omega}\left(\nabla u^{(n)}(x, \tau)\right)^{2} d x .
\end{aligned}
$$

We may obtain similar inequalities for the third and the fourth terms in the right-hand side of (4.11). Now if we apply Cauchy Schwarz inequality to the last term in (4.11) we get

$$
\left|\int_{0}^{\tau} \int_{\Omega} f u_{t}^{(n)} d x d t\right| \leq \frac{1}{2} \int_{0}^{\tau} \int_{\Omega}|f(x, t)|^{2} d x d t+\frac{1}{2} \int_{0}^{\tau} \int_{\Omega}\left|u_{t}^{(n)}(x, t)\right|^{2} d x d t
$$


International Journal of Mathematics and Mathematical Sciences

11

Using the result inequalities in (4.11) and then regrouping the same terms yield

$$
\begin{aligned}
& \int_{\Omega}\left[\left(u_{t}^{(n)}(x, \tau)\right)^{2}+c(1-\varepsilon)\left(\nabla u^{(n)}(x, \tau)\right)^{2}+\left(b-c\left(c(\varepsilon)+k_{1}^{2}|\partial \Omega|\right)\right)\left(u^{(n)}(x, \tau)\right)^{2}\right] d x \\
& \leq \int_{\Omega}\left[\left(u_{t}^{(n)}(x, 0)\right)^{2}+(c+c \varepsilon)\left(\nabla u^{(n)}(x, 0)\right)^{2}+\left(b+c\left(c(\varepsilon)+k_{1}^{2}|\partial \Omega|\right)\right)\left(u^{(n)}(x, 0)\right)^{2}\right] d x \\
& \quad+c\left(c(\varepsilon)+k_{1}^{2}|\partial \Omega|\right) \int_{0}^{\tau} \int_{\Omega}\left(u^{(n)}(x, \mathrm{t})\right)^{2} d x d t \\
& \quad+c \varepsilon \int_{0}^{\tau} \int_{\Omega}\left(\nabla u^{(n)}(x, t)\right)^{2} d x d t \\
& \quad+\int_{0}^{\tau} \int_{\Omega}|f(x, t)|^{2} d x d t+\int_{0}^{\tau} \int_{\Omega}\left|u_{t}^{(n)}(x, t)\right|^{2} d x d t .
\end{aligned}
$$

Choose $\epsilon$ such that $(1-\varepsilon)>0$ and $b-c\left(c(\varepsilon)+k_{1}^{2}|\partial \Omega|\right)>0$ and let

$$
\begin{aligned}
& M=\max \left\{1,(c+c \varepsilon),\left(b+c\left(c(\varepsilon)+k_{1}^{2}|\partial \Omega|\right)\right)\right\}, \\
& m=\min \left\{1-\varepsilon, b-c\left(c(\varepsilon)+k_{1}^{2}|\partial \Omega|\right)\right\}, \quad K_{2}=\frac{M}{m},
\end{aligned}
$$

then (4.14) becomes

$$
\begin{aligned}
& \int_{\Omega}\left[\left(u_{t}^{(n)}(x, \tau)\right)^{2}+\left(\nabla u^{(n)}(x, \tau)\right)^{2}+\left(u^{(n)}(x, \tau)\right)^{2}\right] d x \\
& \leq K_{2}\left[\int_{\Omega}\left(\left(\psi^{(n)}(x)\right)^{2}+\left(\nabla \varphi^{(n)}(x)\right)^{2}+\left(\varphi^{(n)}(x)\right)^{2}\right) d x\right. \\
& \quad+\int_{0}^{\tau} \int_{\Omega}\left(\left|u_{t}^{(n)}(x, t)\right|^{2}+\left(u^{(n)}(x, t)\right)^{2}\left(\nabla u^{(n)}(x, t)\right)^{2}\right) d x d t \\
& \left.\quad+\int_{0}^{T} \int_{\Omega}|f(x, t)|^{2} d x d t\right] .
\end{aligned}
$$

Now, we apply Gronwall Lemma to get

$$
\begin{aligned}
\int_{\Omega} & {\left[\left(u_{t}^{(n)}(x, \tau)\right)^{2}+\left(\nabla u^{(n)}(x, \tau)\right)^{2}+\left(u^{(n)}(x, \tau)\right)^{2}\right] d x } \\
& \leq e^{K_{2} T}\left(\int_{\Omega}\left[\left(\psi^{(n)}(\mathrm{x})\right)^{2}+\left(\nabla \varphi^{(n)}(x)\right)^{2}+\left(\varphi^{(n)}(x)\right)^{2}\right] d x+\|f\|^{2}\right) .
\end{aligned}
$$


Since $\varphi^{(n)}$ and $\psi^{(n)}$ are the orthogonal projections of $\varphi$ and $\psi$, respectively, from the Pythagorean relation we have that $\left\|\varphi^{(n)}\right\|_{W^{1,2}(\Omega)}^{2} \leq\|\varphi\|_{W^{1,2}(\Omega)}^{2}$ and $\left\|\psi^{(n)}\right\|_{L_{2}(\Omega)}^{2} \leq\|\psi\|_{L_{2}(\Omega)}^{2}$. Integrating (4.17) according to $\tau$ on $[0, T]$ yields

$$
\left\|u^{(n)}\right\|_{W^{1,2}(Q)}^{2} \leq T e^{K_{2} T}\left[\|f\|^{2}+\|\psi\|_{L_{2}(\Omega)}^{2}+\|\varphi\|_{W^{1,2}(\Omega)}^{2}\right] .
$$

Consequently, the sequence $\left\{u^{(n)}\right\}$ is bounded.

Remark 4.3. We have proved that the sequence $\left\{u^{(n)}\right\}$ is bounded, so we can extract a subsequence which we denote by $\left\{u^{\left(n_{k}\right)}\right\}$ that is weakly convergent; then we prove that its limit is the desired solution of the problem (1.6)-(1.8).

Lemma 4.4. The limit of the subsequence $\left\{u^{\left(n_{k}\right)}\right\}$ is the solution of the problem (1.6)-(1.8).

Proof. For this we prove that the limit of the subsequence $\left\{u^{\left(n_{k}\right)}\right\}$ satisfies the identity (2.8) for all functions $v=\sum_{i=1}^{n} v_{i}(t) w_{i}(x) \in W_{T}^{1,2}(Q)$. Since the set $S_{n}=\left\{v(x, t)=\sum_{k=1}^{n} v_{k}(t) w_{k}(x)\right.$, $\left.v_{k}(t) \in C^{2}(0, T), v_{k}(T)=0\right\}$ is such that $\cup_{n=1}^{\infty} S_{n}$ is dense in $W_{T}^{1,2}(Q)$, it suffices to prove (2.8) for $v \in S_{n}$. Multiplying (4.3) by the function $v_{i}(t) \in W^{1,2}(0, T), v_{i}(T)=0$, and then taking the sum from $i=0$ to $n$, we obtain

$$
\begin{aligned}
& \int_{\Omega}\left(c \nabla u^{\left(n_{k}\right)} \nabla v+u_{t t}^{\left(n_{k}\right)} v+a u_{t}^{\left(n_{k}\right)} v+b u^{\left(n_{k}\right)} v\right) d x \\
& \quad+c \int_{\partial \Omega} v\left(\int_{\Omega} K(x, \xi) u^{\left(n_{k}\right)}(\xi, t) d \xi\right) d s=\int_{\Omega} f v d x .
\end{aligned}
$$

Integrating by parts on $[0, T]$, we get

$$
\begin{gathered}
\int_{Q}\left(c \nabla u^{\left(n_{k}\right)} \nabla v-u_{t}^{\left(n_{k}\right)} v-a u^{\left(n_{k}\right)} v+b u^{\left(n_{k}\right)} v\right) d x d t+c \int_{0}^{T} \int_{\partial \Omega} v\left(\int_{\Omega} K(x, \xi) u^{\left(n_{k}\right)}(\xi, t) d \xi\right) d s d t \\
=\int_{\Omega} \psi^{\left(n_{k}\right)}(x) v(x, 0) d x+a \int_{\Omega} \varphi^{\left(n_{k} n_{k}\right)}(x) v(x, 0) d x+\int_{Q} f v d x d t .
\end{gathered}
$$

Denote the weak limit of the subsequence $\left\{u^{\left(n_{k}\right)}\right\}$ by $u$. When $k$ tends to infinity, we get

$$
\int_{0}^{T} \int_{\partial \Omega} v\left(\int_{\Omega} K(x, \xi) u^{\left(n_{k}\right)}(\xi, t) d \xi\right) d s d t \rightarrow \int_{0}^{T} \int_{\partial \Omega} v\left(\int_{\Omega} K(x, \xi) u(\xi, t) d \xi\right) d s d t
$$


Indeed, using Cauchy Schwarz inequality then $\varepsilon$-Cauchy inequality, we obtain

$$
\begin{aligned}
& \left|\int_{0}^{T} \int_{\partial \Omega} v\left(\int_{\Omega} K(x, \xi) u^{\left(n_{k}\right)}(\xi, t) d \xi\right) d s d t-\int_{0}^{T} \int_{\partial \Omega} v\left(\int_{\Omega} K(x, \xi) u(\xi, t) d \xi\right) d s d t\right| \\
& \leq k_{1}|\partial \Omega|\left(\int_{0}^{T} \int_{\partial \Omega}|v|^{2} d s d t\right)^{1 / 2} \\
& \quad \times\left(\int_{0}^{T} \int_{\Omega}\left|u^{\left(n_{k}\right)}(\xi, t)-u(\xi, t)\right|^{2} d \xi d s d t\right)^{1 / 2} \rightarrow 0
\end{aligned}
$$

and then by passing to the limit in (4.20) we get that the limit $u$ satisfies(2.8).

Example 4.5. Now we present an example to demonstrate the applications of the results established in the earlier sections. We consider the following:

$$
\frac{\partial^{2} u}{\partial t^{2}}+\frac{\partial u}{\partial t}+u-\frac{\partial^{2} u}{\partial x^{2}}=\sin x(\sin t+\cos t), \quad 0<t \leq T, x \in(0, \pi)
$$

with the initial conditions

$$
u(x, 0)=0, \quad \frac{\partial u}{\partial t(x, 0)}=\sin x, \quad x \in(0, \pi)
$$

and the nonlocal boundary condition

$$
\frac{\partial u}{\partial x}(\pi, t)-\frac{\partial u}{\partial x}(0, t)-\int_{0}^{\pi} u(s, t) d s=0
$$

The results of the earlier sections guarantee the existence and uniqueness of a solution. It may be noted that $u(x, t)=\sin x \sin t$ is the unique solution of (4.23)-(4.25).

\section{Acknowledgment}

The authors thank the referees for their helpful suggestions and corrections to improve the paper.

\section{References}

[1] J. R. Cannon, "The solution of the heat equation subject to the specification of energy," Quarterly of Applied Mathematics, vol. 21, no. 3, pp. 155-160, 1963.

[2] G. Avalishvili and D. Gordeziani, "On one class of spatial nonlocal problems for some hyperbolic equations," Georgian Mathematical Journal, vol. 7, no. 3, pp. 417-425, 2000.

[3] S. A. Beilin, "Existence of solutions for one-dimensional wave equations with nonlocal conditions," Electronic Journal of Differential Equations, vol. 76, pp. 1-8, 2001.

[4] J. R. Cannon and J. van der Hoek, "Diffusion subject to the specification of mass," Journal of Mathematical Analysis and Applications, vol. 115, no. 2, pp. 517-529, 1986. 
[5] J. R. Cannon, S. Pérez Esteva, and J. van der Hoek, "A Galerkin procedure for the diffusion equation subject to the specification of mass," SIAM Journal on Numerical Analysis, vol. 24, no. 3, pp. 499-515, 1987.

[6] D.-Q. Dai and Y. Huang, "Remarks on a semilinear heat equation with integral boundary conditions," Nonlinear Analysis, vol. 67, no. 2, pp. 468-475, 2007.

[7] N. I. Ionkin, "The solution of a certain boundary value problem of the theory of heat conduction with a nonclassical boundary condition," Differents nye Uravneniya, vol. 13, no. 2, pp. 294-304, 1977.

[8] L. I. Kamynin, "A boundary-value problem in the theory of heat conduction with non-classical boundary conditions," Žurnal Vyčislite nŏ Matematiki i Matematičeskoŭ Fiziki, vol. 4, pp. 1006-1024, 1964.

[9] A. I. Kozhanov and L. S. Pulkina, "On the solvability of boundary value problems with a nonlocal boundary condition of integral form for multidimensional hyperbolic equations," Differents nye Uravneniya, vol. 42, no. 9, pp. 1166-1179, 2006.

[10] L. S. Pulkina, "A non-local problem with integral conditions for hyperbolic equations," Electronic Journal of Differential Equations, vol. 45, pp. 1-6, 1999.

[11] W. A. Day, "Extensions of a property of the heat equation to linear thermoelasticity and other theories," Quarterly of Applied Mathematics, vol. 40, no. 3, pp. 319-330, 1982.

[12] G. Fairweather and J. C. López-Marcos, "Galerkin methods for a semilinear parabolic problem with nonlocal boundary conditions," Advances in Computational Mathematics, vol. 6, no. 3-4, pp. 243-262, 1996.

[13] V. Buda, R. Ciegis, and M. Sapagovas, "A model of multiple diffusion from a limited source," Application to Differential Equations, vol. 38, pp. 9-14, 1986.

[14] S. A. Beilin, "On a mixed nonlocal problem for a wave equation," Electronic Journal of Differential Equations, vol. 103, pp. 1-10, 2006.

[15] L. S. Pulkina, "A nonlocal problem with an integral condition of the first kind for a multidimensional hyperbolic equation," Rossiǔskaya Akademiya Nauk, vol. 416, no. 5, pp. 597-599, 2007.

[16] A. Bouziani, N. Merazga, and S. Benamira, "Galerkin method applied to a parabolic evolution problem with nonlocal boundary conditions," Nonlinear Analysis, vol. 69, no. 5-6, pp. 1515-1524, 2008.

[17] D. Bahuguna, S. Abbas, and J. Dabas, "Partial functional differential equation with an integral condition and applications to population dynamics," Nonlinear Analysis: Theory, Methods $\mathcal{E}$ Applications, vol. 69, no. 8, pp. 2623-2635, 2008.

[18] J. Dabas and D. Bahuguna, "An integro-differential parabolic problem with an integral boundary condition," Mathematical and Computer Modelling, vol. 50, no. 1-2, pp. 123-131, 2009.

[19] A. Guezane-Lakoud and D. Belakroum, "Rothe's method for a telegraph equation with integral conditions," Nonlinear Analysis, vol. 70, no. 11, pp. 3842-3853, 2009.

[20] N. Merazga and A. Bouziani, "On a time-discretization method for a semilinear heat equation with purely integral conditions in a nonclassical function space," Nonlinear Analysis: Theory, Methods $\mathcal{E}$ Applications, vol. 66, no. 3, pp. 604-623, 2007.

[21] N. Merazga and A. Bouziani, "Rothe time-discretization method for a nonlocal problem arising in thermoelasticity," Journal of Applied Mathematics and Stochastic Analysis, no. 1, pp. 13-28, 2005.

[22] D. Bahuguna and S. Agarwal, "Approximations of solutions to neutral functional differential equations with nonlocal history conditions," Journal of Mathematical Analysis and Applications, vol. 317, no. 2, pp. 583-602, 2006.

[23] B. G. Pachpatte, Inequalities for Differential and Integral Equations, vol. 197 of Mathematics in Science and Engineering, Academic Press, San Diego, Calif, USA, 1998. 


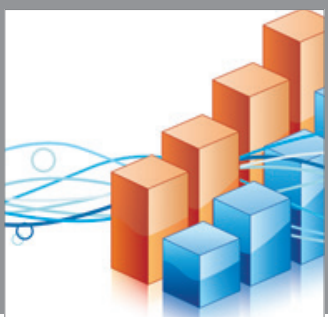

Advances in

Operations Research

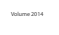

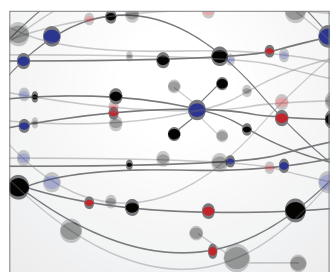

\section{The Scientific} World Journal
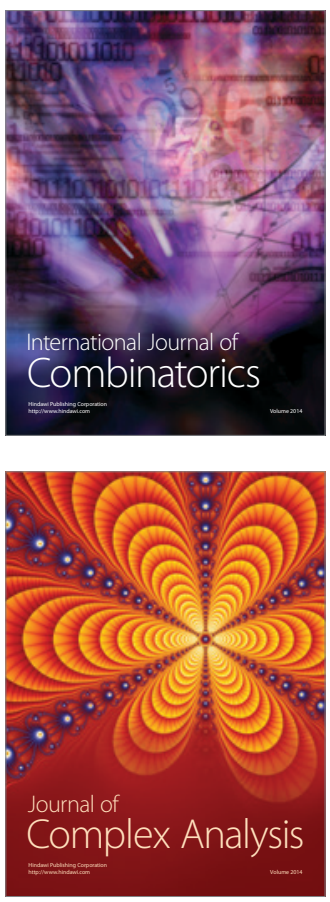

International Journal of

Mathematics and

Mathematical

Sciences
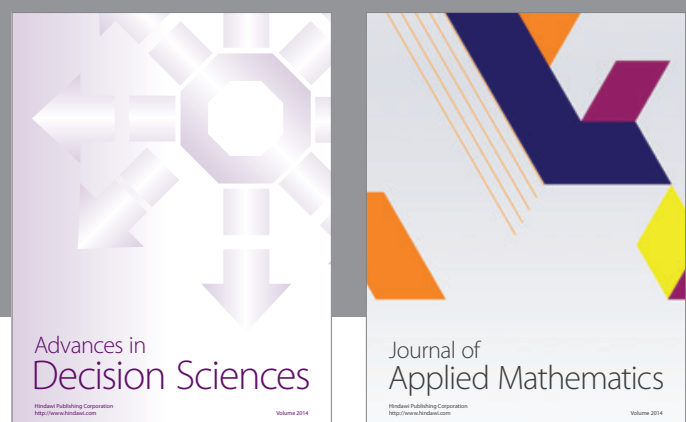

Journal of

Applied Mathematics
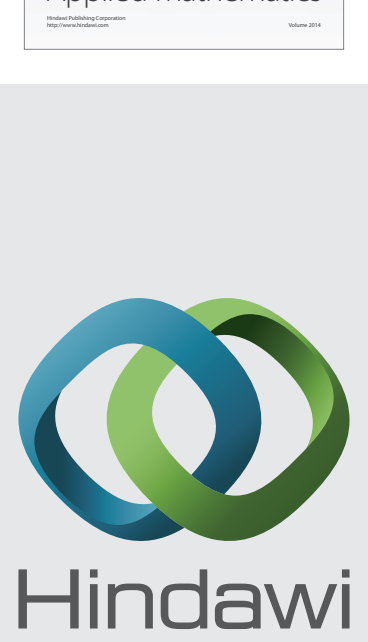

Submit your manuscripts at http://www.hindawi.com
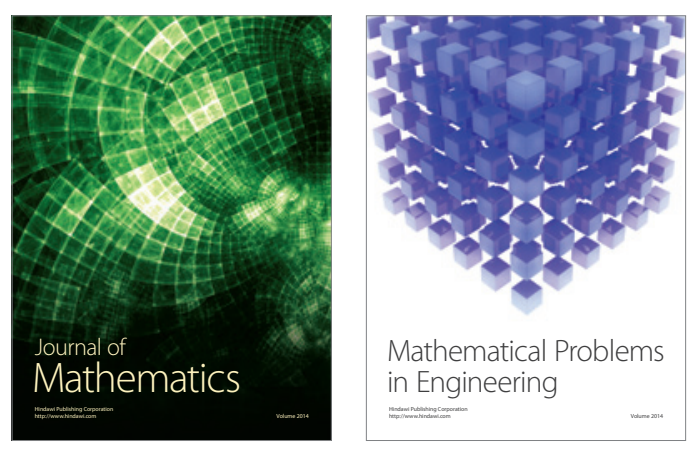

Mathematical Problems in Engineering
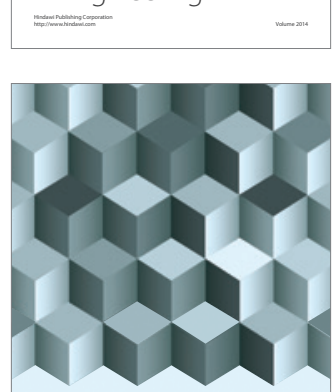

Journal of

Function Spaces
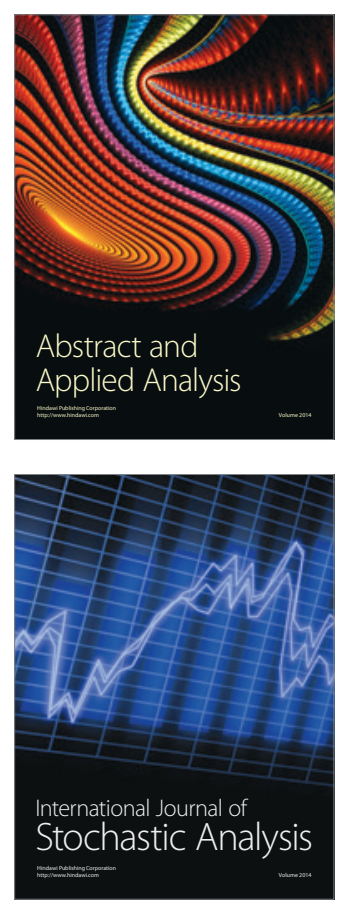

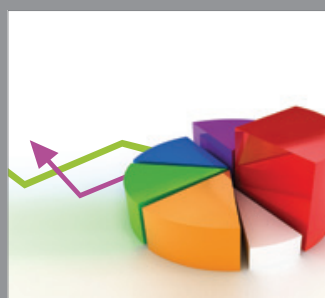

ournal of

Probability and Statistics

Promensencen
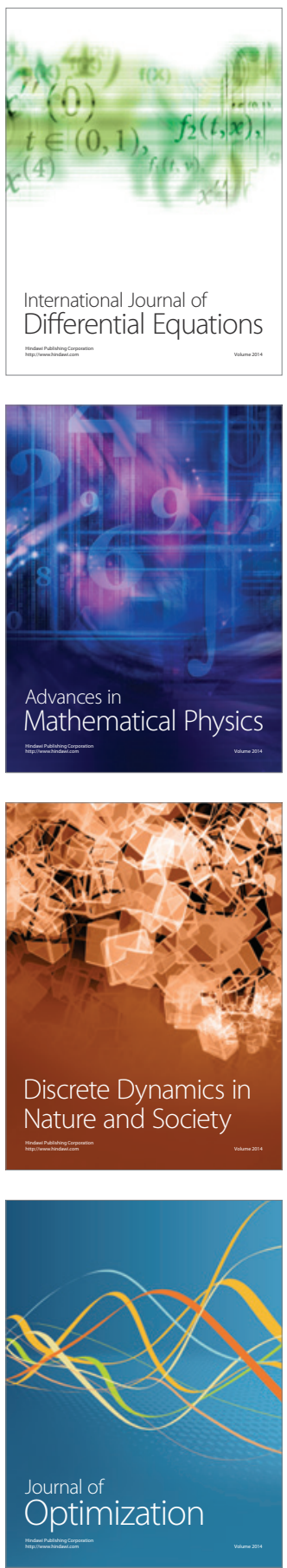